\title{
On Minimal and Maximal Regular Open Sets
}

\author{
Hisham Mahdi*, Fadwa Nasser \\ Department of Mathematics, Islamic University of Gaza, PO Box 108, Gaza, Palestine
}

Copyright (c) 2017 by authors, all rights reserved. Authors agree that this article remains permanently open access under the terms of the Creative Commons Attribution License 4.0 International License

\begin{abstract}
The purpose of this paper is to investigate the concepts of minimal and maximal regular open sets and their relations with minimal and maximal open sets. We study several properties of such concepts in a semi-regular space. It is mainly shown that if $X$ is a semi-regular space, then $m_{i} O(X)=m_{i} R O(X)$. We introduce and study new type of sets called minimal regular generalized closed. A special interest type of topological space called $r T_{\text {min }}$ space is studied and obtain some of its basic properties.
\end{abstract}

Keywords Minimal Open, Minimal Regular Open, Maximal Regular Open, Semi-regular Space, Minimal Regular Generalized Closed

\section{Introduction and Preliminaries}

A subset $A$ of a topological space $(\mathrm{X}, \tau)$ is called a semi-open [14] (resp. a preopen [1], an $\alpha$-open [16]) set if $A \subseteq C l(\operatorname{Int}(A))$ (resp. $A \subseteq \operatorname{Int}(C l(A)), A \subseteq$ $\operatorname{Int}(C l(\operatorname{Int}(A))))$. It is called a semi-closed [19] (resp. a preclosed [13], an $\alpha$-closed [8] ) set if $A^{c}$ is semi-open ( resp. preopen, $\alpha$-open). The family of all semi-open (resp. preopen, $\alpha$-open) sets is denoted by $S O(X)$ (resp. $P O(X)$, $\left.\tau_{\alpha}\right)$. A subset $A$ of a topological space $X$ is said to be a regular open set [10] if $A=\operatorname{Int}(C l(A))$. It is called a regular closed if $A^{c}$ is a regular open. The family of all regular-open (resp. regular-closed) sets is denoted by $R O(X, \tau)$ or simply $R O(X)$ (resp. $R C(X, \tau)$ or simply $R C(X)$ ). In [10], it was shown that the regularly open sets of a space $(\mathrm{X}, \tau)$ is a base for a topology $\tau_{s}$ on $X$ coarser than $\tau$. The space $\left(\mathrm{X}, \tau_{s}\right)$ was called the semi-regularization space of $(\mathrm{X}, \tau)$. The space $(\mathrm{X}, \tau)$ is semi-regular if and only if the regularly open sets of $(\mathrm{X}, \tau)$ is a base for $\tau$; that is, $\tau=\tau_{s}$. For a space $(\mathrm{X}, \tau)$, the regularly open sets of $(\mathrm{X}, \tau)$ equal the regularly open sets of $\left(\mathrm{X}, \tau_{s}\right)$. Hence, the semi-regularization process generates at most one new topology. Thus $\left(\tau_{s}\right)_{s}=\tau_{s}$ [10]. A point $x \in X$ is said to be a $\delta$-cluster point of a subset $A$ if $A \cap U \neq \phi$ for every regular open set $U$ containing $x$.
The set of all $\delta$-cluster points of $A$ is called the $\delta$-closure of $A$, and denoted by $C l_{\delta}(A)$. A subset $A$ is called $\delta$-closed if $A=C l_{\delta}(A)$. The complement of a $\delta$-closed set is called a $\delta$-open. A space $X$ is said to be a locally finite if each of its elements is contained in a finite open set.

Theorem 1.1. Let $(X, \tau)$ be a topological space and $C(\tau)$ the collection of closed sets in $X$. Then

$$
\begin{aligned}
R O(X, \tau)= & \{\operatorname{Int}(C l(O)): O \in \tau\}[3] \\
= & \{\operatorname{Ext}(O): O \in \tau\}[2], \text { where } \operatorname{Ext}(O) \\
& \text { denote the set of all exterior points of } O \\
= & \{\operatorname{Int}(C): C \in C(\tau)\} \cdot[5]
\end{aligned}
$$

And

$$
\begin{aligned}
R C(X, \tau) & =\{C l(\operatorname{Int}(C)): C \in C(\tau)\} \\
& =\{C l(U): U \in R O(X, \tau)\} \\
& =\{C l(O): O \in \tau\} \\
& =\left\{C l(V): V \in \tau_{s}\right\}[4] .
\end{aligned}
$$

Theorem 1.2. [9] Let $(X, \tau)$ be a topological space and $A$ a nonempty subspace of $X$. If $A$ is open or dense in $X$, then $R O\left(A, \tau_{A}\right)=\{V \cap A: V \in R O(X, \tau)\}$.

Theorem 1.3. [12] If $A$ and $B$ are disjoint open sets in $(\mathrm{X}, \tau)$, then $\operatorname{Int}(C l(A))$ and $\operatorname{Int}(C l(B))$ are disjoint open sets in $\left(\mathrm{X}, \tau_{s}\right)$ containing $A$ and $B$ respectively.

A proper nonempty open (resp. closed) subset $U$ of $X$ is said to be a minimal open (resp. a minimal closed) set [6] if any open (resp. closed) set which is contained in $U$ is $\phi$ or $U$. A proper nonempty open (resp. closed) subset $M$ of $X$ is said to be a maximal open (resp. a maximal closed) set [7] if any open (resp. closed) set which contains $M$ is $X$ or $M$. The collection of all minimal open (resp. maximal open, minimal closed, maximal closed) sets is denoted by $m_{i} O(X)$ (resp. $M_{a} O(X), m_{i} C(X), M_{a} C(X)$ ).

Lemma 1.4. [6] Let $X$ be a topological space and $U$ a minimal open set. 
(a) If $W$ is an open set such that $U \cap W \neq \phi$, then $U \subseteq W$.

(b) If $V$ is a minimal open set such that $U \cap V \neq \phi$, then $U=V$.

Theorem 1.5. [6] Let $U$ be a nonempty proper open set in a topological space $X$. Then the following three conditions are equivalent :

(1) $U$ is a minimal open set.

(2) $U \subseteq C l(S)$ for any nonempty subset $S$ of $U$.

(3) $C l(S)=C l(U)$ for any nonempty subset $S$ of $U$.

Theorem 1.6. [6] Let $U$ be a minimal open set in $X$. Then any nonempty subset $S$ of $U$ is preopen set .

Theorem 1.7. [7] Let $U$ be a maximal open set. Then, $C l(U)=X$ or $C l(U)=U$.

Definition 1.8. A subset $A$ of $X$ is said to be:

(a) a generalized minimal closed [17] (briefly. g-minclosed) if $C l(A) \subseteq U$ whenever $A \subseteq U$ and $U$ is a minimal open set.

(b) a minimal generalized closed [18] (briefly. min-gclosed) if $A$ is contained in a minimal open set $U$ such that $C l(A) \subseteq U$.

(c) a regular generalized closed [15] (briefly. regular gclosed) if $C l(A) \subseteq U$ whenever $A \subseteq U$ and $U$ is a regular open set.

Definition 1.9. A nonempty proper regular open set A of a topological space $(\mathrm{X}, \tau)$ is said to be:

a) a minimal regular open set [20] if any regular open set contained in $A$ is $A$ or $\phi$ and a minimal regular closed set [11] if any regular closed set contained in $A$ is $A$ or $\phi$.

b) a maximal regular open set [11] if any regular open set contains $A$ is $X$ or $A$ and a maximal regular closed set [20] if any regular closed set contains $A$ is $X$ or $A$.

The collection of all minimal regular open (resp. minimal regular closed, maximal regular open, maximal regular closed) sets in a topological space $(\mathrm{X}, \tau)$ is denoted by $m_{i} R O(X, \tau)$ (resp. $m_{i} R C(X, \tau), M_{a} R O(X, \tau)$, $M_{a} R C(X, \tau)$ ).

Theorem 1.10. Let $X$ be a topological space and $F \subseteq X$. Then $F$ is a minimal regular open (resp. a minimal regular closed) set if and only if $X \backslash F$ is a maximal regular closed (resp. a maximal regular open) set.

Proof. Let $V$ be a regular closed such that $X \backslash F \subseteq V$, then $X \backslash V \subseteq F$. This implies that $X \backslash V=\phi$ or $X \backslash V=F$; that is, $V=X$ or $V=X \backslash F$.
Example 1.11. Let $X=\{a, b, c, d\}$ with a topology $\tau=$ $\{\phi, X,\{a\},\{b\},\{c\},\{a, b\},\{a, c\},\{b, c\},\{b, d\},\{a, b, c\}$, $\{a, b, d\},\{b, c, d\}\}$. Then $R O(X, \tau)=\{\phi, X,\{a\},\{c\}$, $\{a, c\},\{b, d\},\{a, b, d\},\{b, c, d\}\}$. The set $\{b\}$ is minimal open, but not minimal regular open and the set $\{b, d\}$ is minimal regular open, but not minimal open. In addition, the set $\{a, c\}$ is maximal regular open, but not maximal open and the set $\{a, b, c\}$ is maximal open, but not maximal regular open.

Remark 1.12. If $A$ is a regular open and a minimal open (resp. a maximal open), then $A$ is minimal regular open (resp. maximal regular open).

Lemma 1.13. [11] Let $X$ be a topological space and $U$ a minimal regular open set.

(a) If $W$ is a regular open set such that $U \cap W \neq \phi$, then $U \subseteq W$.

(b) If $V$ is a minimal regular open set such that $U \cap V \neq \phi$, then $U=V$.

Remark 1.14. If $U$ is a maximal regular open in $X$ and $W$ is a regular open, then we may have that $U \cup W \neq X$ and $W \nsubseteq U$. Thus, if $U$ and $V$ are two different maximal regular open sets, we may have that $U \cup V \neq X$.

Example 1.15. Let $X=\{1,2,3,4,5\}$ with a base $\beta=\{\{1\},\{2\},\{3\},\{5\},\{3,4,5\}\}$. Then $R O(X)=$ $\{\phi, X,\{1\},\{2\},\{3\},\{5\},\{1,2\},\{1,3\},\{1,5\},\{2,3\},\{2,5\}$, $\{3,4,5\},\{1,2,5\},\{1,2,3\},\{2,3,4,5\},\{1,3,4,5\}\}$. So

$\{1,2,5\}$ and $\{1,2,3\}$ are two maximal regular open sets, but neither $\{1,2,5\} \cup\{1,2,3\}=X$ nor $\{1,2,5\}=\{1,2,3\}$.

Theorem 1.16. Let $V$ be a nonempty finite regular open set in a topological space $X$. Then there exists at least one ( $f$ nite) minimal regular open set $U$ such that $U \subseteq V$.

Proof. If $V$ is not minimal regular open set, then there exists a (finite) regular open set $V_{1} \neq \phi$ such that $V_{1} \subset V\left(:=V_{1} \subseteq\right.$ $V$ and $V_{1} \neq V$ ). If $V_{1}$ is a minimal regular open set, set $U=V_{1}$. Otherwise there exists a (finite) regular open set $V_{2}$ such that $\phi \neq V_{2} \subset V_{1} \subset V$. Continuing this process only finitely many, we get a minimal regular open set $U=V_{n}$ for some positive integer $n$.

Corollary 1.17. Let $X$ be a locally finite semi-regular space. If $V$ is a nonempty open set, then there exists at least one (finite) minimal regular open set $U$ such that $U \subseteq V$.

Proof. Let $V$ be a nonempty open set with $x \in V$. Since $X$ is locally finite space, there exists a finite open set $V_{x}$ such that $x \in V_{x}$. As $X$ semi-regular, there exists a regular open set $A$ such that $x \in A \subseteq V_{x} \cap V$. By Theorem 1.16, there exists a minimal regular open set $U$ such that $U \subseteq A$. 


\section{More on Minimal and maximal Reg- ular Open Sets}

Theorem 2.1. If $A$ is a minimal open set in a space $X$ such that $A$ is not dense in $X$, then $\operatorname{Int}(C l(A))$ is a minimal regular open.

Proof. Since $A$ is a nonempty open set and not dense, then $\phi \neq A \subseteq \operatorname{Int}(C l(A)) \neq X$; that is, $\operatorname{Int}(C l(A))$ is a nonempty proper regular open. Let $V$ be a nonempty regular open set such that $V \subseteq \operatorname{Int}(C l(A))$. Then $\operatorname{Int}(C l(V)) \subseteq$ $\operatorname{Int}(C l(A))$. If $V \cap A=\phi$, then by Theorem 1.3, $\operatorname{Int}(C l(V)) \cap \operatorname{Int}(C l(A))=\phi$, which is a contradiction. So, $V \cap A \neq \phi$. By Lemma 1.4 part $(a), A \subseteq V$ and so $\operatorname{Int}(C l(A)) \subseteq \operatorname{Int}(C l(V))$.

Remark 2.2. From Theorem 2.1, any non dense minimal open set $A$, there exists a minimal regular open set $U$ such that $A \subseteq U$.

Theorem 2.3. If $C$ is a closed set contained properly in a minimal regular open set $U$, then $\operatorname{Int}(C)=\phi$.

Proof. $\operatorname{Int}(C)$ is a regular open in $U$.

Theorem 2.4. Let $U$ be a nonempty proper regular open set. Then, the following three conditions are equivalent:

(1) $U$ is a minimal regular open.

(2) $U \subseteq C l(A)$, where $A \subseteq U$ and $\operatorname{Int}(A) \neq \phi$.

(3) $C l(U)=C l(A)$, where $A \subseteq U$ and $\operatorname{Int}(A) \neq \phi$.

Proof. $(1 \Rightarrow 2)$ Since $\phi \neq \operatorname{Int}(A) \subseteq \operatorname{Int}(C l(A)) \subseteq$ $\operatorname{Int}(C l(U))=U$, then $\operatorname{Int}(C l(A))=U$. Therefore, $U \subseteq C l(A)$.

$(2 \Rightarrow 3)$ Since $A \subseteq U, U \subseteq C l(A) \subseteq C l(U)$. Therefore, $C l(U)=C l(A)$.

$(3 \Rightarrow 1)$ Let $B$ be a nonempty regular open set such that $B \subseteq$ $U$, then $\phi \neq \operatorname{Int}(B)=B \subseteq U$, so $C l(U)=C l(B)$. This implies that $B=\operatorname{Int}(C l(B))=\operatorname{Int}(C l(U))=U$.

Corollary 2.5. If $U$ is a minimal regular open and $A \subseteq U$ such that $\operatorname{Int}(A) \neq \phi$, then $A$ is preopen.

Remark 2.6. The condition of being $\operatorname{Int}(A) \neq \phi$ is necessary in Theorem 2.4 and Corollary 2.5 as shown in the following example:

Example 2.7. In Example 1.11, the set $\{b, d\}$ is minimal regular open, $\{d\} \subseteq\{b, d\}$ and $\operatorname{Int}(\{d\})=\phi$, but $\{b, d\} \nsubseteq$ $\{d\}=C l(\{d\})$. In Addition, the set $\{d\}$ is not preopen.

Theorem 2.8. Let $A$ be a nonempty subspace of $X$ and $U$ a regular open set in $A$ and a regular open in $X$. If $U$ is a minimal regular open in $A$, then $U$ is a minimal regular open in $X$.
Proof. $U$ is a nonempty proper regular open set in $X$. Let $S \subseteq U$ such that $\operatorname{Int}_{X}(S) \neq \phi$. Then, $\phi \neq \operatorname{Int}_{X}(S)=$ $\operatorname{Int}_{X}(S) \cap A \subseteq \operatorname{Int}_{A}(S)$. So by Theorem 2.4, $U \subseteq$ $C l_{A}(S)=C l_{X}(S) \cap A \subseteq C l_{X}(S)$. Thus, the result follows by Theorem 2.4 .

Theorem 2.9. Let $A$ be an open subspace of $X$ and $G$ a minimal regular open set in $X$. If $G \cap A \neq \phi$ and $A \nsubseteq G$, then $G \cap A$ is a minimal regular open in $A$.

Proof. By Theorem 1.2, $G \cap A$ is a regular open in $A$. Let $S \subseteq G \cap A$ such that $\operatorname{Int}_{A}(S) \neq \phi$. Then $\operatorname{Int}_{X}(S) \neq \phi$, and by Theorem 2.4, $G \subseteq C l_{X}(S)$. Thus, $G \cap A \subseteq C l_{X}(S) \cap$ $A=C l_{A}(S)$. By Theorem 2.4, $G \cap A$ is a minimal regular open in $A$.

Remark 2.10. Theorem 2.9 need not be true if $A$ is not open subspace as shown in the following example:

Example 2.11. Let $X=\{1,2,3,4\}$ with the topology $\tau=\{\phi, X,\{1\},\{2\},\{4\},\{1,2\},\{1,4\},\{2,4\},\{1,2,4\}$, $\{1,2,3\}\}$. Then $R O(X, \tau)=\{\phi, X,\{1\},\{2\},\{4\},\{1,4\}$, $\{2,4\},\{1,2,3\}\}$. Let $A=\{2,3,4\}$. Then $\tau_{A}=\{\phi, A,\{2\},\{2,3\},\{4\},\{2,4\}\}$. So, $R O(A)=$ $\{\phi, A,\{2,3\},\{4\}\}$. The set $\{2\}$ is a minimal regular open in $X$ and $\{2\} \cap A \neq \phi$, but $\{2\} \cap A=\{2\}$ is not minimal regular open set in $A$.

Remark 2.12. If $A$ is a regular closed, then $\operatorname{Int}(A)$ is a regular open.

Theorem 2.13. Let $A$ be a minimal regular open. If $A$ is closed set, then $A$ is minimal regular closed.

Proof. Since $A$ is regular open and closed, then $A$ is regular closed. Suppose that $B$ is a regular closed such that $B \subseteq A$. Then $\operatorname{Int}(B)$ is a regular open and $\operatorname{Int}(B) \subseteq B \subseteq A$. As $A$ is minimal regular open, $\operatorname{Int}(B)=\phi$ or $\operatorname{Int}(B)=A$. Then either $C l(\operatorname{Int}(B))=\phi$ or $C l(\operatorname{Int}(B))=C l(A)=A$. Since $C l(\operatorname{Int}(B))=B$, we get $B=\phi$ or $B=A$.

Theorem 2.14. Let $X$ be a topological space and $U$ a proper nonempty regular open set. Then the following three conditions are equivalent:

(1) $U$ is a minimal regular open.

(2) $U \subseteq C l_{\delta}(S), \forall S \subseteq U$ and $S \neq \phi$.

(3) $C l_{\delta}(U)=C l_{\delta}(S), \forall S \subseteq U$ and $S \neq \phi$.

Proof. $(1 \Rightarrow 2)$ Let $x \in U$ and $W$ a regular open set containing $x$, then $\phi \neq S=S \cap U \subseteq S \cap W$. This implies that $x \in C l_{\delta}(S)$.

$(2 \Rightarrow 3)$ Since $S \subseteq U$, then $C l_{\delta}(S) \subseteq C l_{\delta}(U)$. So $C l_{\delta}(U)=C l_{\delta}(S)$.

$(3 \Rightarrow 1)$ If $U$ is not minimal regular open, then there exists a nonempty regular open $V$ such that $V \subset U$. So, there exists an element $x \in U$ such that $x \notin V$. Then $C l_{\delta}(\{x\}) \subseteq X \backslash V$. Hence $C l_{\delta}(\{x\}) \neq C l_{\delta}(U)$ which is a contradiction. 
Corollary 2.15. Let $M$ be a maximal regular closed. If $A$ is a super set of $M$, then $C l_{\delta}(A)=X$.

Proof. Let $S$ be a nonempty subset of $X \backslash M$ such that $A=$ $M \cup S$. As $X \backslash M$ is minimal regular open, by Theorem 2.14, $X \backslash M \subseteq C l_{\delta}(S)$. So $C l_{\delta}(A)=C l_{\delta}(M) \cup C l_{\delta}(S) \supseteq M \cup$ $X \backslash M=X$.

Theorem 2.16. If $C$ is a proper closed set contains a maximal regular open set $M$, then $\operatorname{Int}(C)=M$.

Proof. Since $M \subseteq \operatorname{Int}(C) \subseteq C \neq X$ and $\operatorname{Int}(C)$ is a regular open, then $\operatorname{Int}(C)=M$.

Theorem 2.17. If $M$ is a nonempty proper regular open set such that there is no proper closed set contains $M$ properly, then $M$ is a maximal regular open.

Proof. Let $V$ be a regular open set such that $M \subseteq V \subseteq$ $C l(V)$. Then as $C l(V)$ is a closed set, $C l(V)=M$ or $C l(V)=X$. Since $V=\operatorname{Int}(C l(V))$, we get $V=$ $\operatorname{Int}(M)=M$ or $V=\operatorname{Int}(X)=X$.

Theorem 2.18. A nonempty proper regular open set $M$ is a maximal regular open if and only if $\operatorname{Int}(C)=M$ for any proper closed set $C$ contains $M$.

Proof. If $M$ is a maximal regular open set, then the result follows from Theorem 2.16. Conversely, if $M \subseteq V \subseteq C l(V)$ where $V$ is regular open set such that $V \neq X$, then $C l(V)$ is closed set and $C l(V) \neq X$ (Otherwise $V=\operatorname{Int}(C l(V))=$ $X)$. By given, $M=\operatorname{Int}(C l(V))=V$.

Theorem 2.19. If $A$ is a maximal open set in a topological space $X$, then exactly one of the following holds:

(1) A is a maximal regular open.

(2) A is dense in $X$.

Proof. By Remark 1.12, it suffices to prove that if $A$ is not dense, then $A$ is regular open. If $A$ is not dense, then $A \subseteq$ $C l(A) \subset X$. Then $A \subseteq \operatorname{Int}(C l(A)) \subset X$. As $A$ is maximal open, we get $A=\operatorname{Int}(C l(A))$.

\section{Semi-Regular Spaces and Semi- Regularization}

Theorem 3.1. Let $X$ be a semi-regular space. Then, $U$ is a minimal regular open set if and only if $U$ is a minimal open set; that is, $m_{i} O(X)=m_{i} R O(X)$.

Proof. Let $U \in m_{i} O(X)$ with $x \in U$. Then there exists a regular open set $W$ such that $x \in W \subseteq U$. Since $W$ is a nonempty open set, $W=U$; that is, $U$ is regular open set. So by Remark 1.12, $U$ is a minimal regular open set. Conversely, let $U \in m_{i} R O(X)$ and $G$ a nonempty open set such that $G \subseteq U$. Let $x \in G$, then there exists a regular open set $A$ such that $x \in A \subseteq G \subseteq U$ and so $A=U$. This implies that $G=U$.
Remark 3.2. If $X$ is not semi-regular space, then $M_{i} O(X)$ and $M_{i} R O(X)$ are, in general, independent as shown in Example 1.11. Moreover, $M_{a} O(X)$ and $M_{a} R O(X)$ are independent even if $X$ is semi-regular space. See the following example:

Example 3.3. In Example 1.15, the set $\{1,2,3,5\}$ is a maximal open, but not maximal regular open and the set $\{1,2,5\}$ is a maximal regular open, but not maximal open. However, the space $(X, \tau)$ is semi-regular.

Corollary 3.4. Let $\left(X, \tau_{s}\right)$ be the semi-regularization space of a topological space $(X, \tau)$. Then, $m_{i} O\left(X, \tau_{s}\right)$ $=m_{i} R O\left(X, \tau_{s}\right)$.

Corollary 3.5. Let $X$ be a semi-regular space and $A \subseteq X$. If $A$ is contained properly in a minimal regular open set $U$, then $\operatorname{Int}(A)=\phi$

Corollary 3.6. Let $X$ be a semi-regular space. If $U$ is a minimal regular open set, then any nonempty subset $S$ of $U$ is preopen set.

Proof. Follows from Theorem 3.1 and Theorem 1.6.

Corollary 3.7. Let $X$ be a semi-regular space and $U$ a nonempty proper regular open set, then the following three conditions are equivalent:

(1) $U$ is a minimal regular open.

(2) $U \subseteq C l(S)$ for any nonempty subset $S$ of $U$.

(3) $C l(S)=C l(U)$ for any nonempty subset $S$ of $U$.

Proof. The result follows directly from Theorem 3.1 and Theorem 1.5.

Corollary 3.8. Let $X$ be a semi-regular space. If $U$ is a maximal regular closed and $M$ a proper super set of $U$, then $C l(M)=X$.

Proof. Let $S$ be a nonempty subset of $X \backslash U$ such that $M=$ $U \cup S$. As $X \backslash U$ is minimal regular open, by Corollary 3.7, $X \backslash U \subseteq C l(S)$. Hence $C l(M)=C l(U) \cup C l(S) \supseteq U \cup$ $X \backslash U=X$.

Remark 3.9. The condition of being $X$ semi-regular is necessary in Corollary 3.5, Corollary 3.6, Corollary 3.7 and Corollary 3.8 as shown in the following example:

Example 3.10. In Example 1.11, $X$ is not semi-regular. The set $\{b, d\}$ is minimal regular open and $\{b\} \subset\{b, d\}$, but $\operatorname{Int}(\{b\})=\{b\} \neq \phi$. In Addition, the set $\{d\} \subseteq\{b, d\}$, but The set $\{d\}$ is not preopen. Moreover, $\{b, d\} \nsubseteq C l(\{d\})=$ $\{d\}$; that is, $C l(\{b, d\})=\{b, d\} \neq\{d\}=C l(\{d\})$. The set $\{a, c\}$ is maximal regular closed and $\{a, c, d\} \supset\{a, c\}$, but $C l(\{a, c, d\})=\{a, c, d\} \neq X$.

Theorem 3.11. Let $\left(X, \tau_{s}\right)$ be the semi-regularization space of a topological space $(X, \tau)$. Then, $m_{i} R O(X, \tau)$ $=m_{i} R O\left(X, \tau_{s}\right)$ and $M_{a} R O(X, \tau)=M_{a} R O\left(X, \tau_{s}\right)$.

Proof. Direct from the fact that $R O(X, \tau)=R O\left(X, \tau_{s}\right)$. 


\section{Generalized Minimal Regular Closed Sets}

Definition 4.1. A subset $A$ of a topological space $X$ is called:

(a) a generalized minimal regular closed (briefly. g-mr closed) if $C l(A) \subseteq U$ whenever $A \subseteq U$ and $U$ is a minimal regular open.

(b) a minimal regular generalized closed (briefly. mr-gclosed) if $A$ is contained in a minimal regular open set $U$ and $C l(A) \subseteq U$.

Remark 4.2. Using Lemma $1.13(b)$,

(1) If $A$ is mr-g-closed, then there exists a unique minimal regular open set $U$ such that $C l(A) \subseteq U$. This implies that $A$ is g-mr closed. That is every mr-g-closed set is also g-mr closed set.

(2) Let $A \subseteq X$. If there is no minimal regular open set $U$ such that $A \subseteq U$, then $A$ is g-mr closed, but not mr-gclosed.

Example 4.3. In Example 1.11, $\{a, b\}$ is g-mr closed, but not $\mathrm{mr}$-g-closed. The set $\{b\}$ is both $\mathrm{mr}$-g-closed and $\mathrm{g}$ - $\mathrm{mr}$ closed.

Remark 4.4. The class of g-mr closed sets and the class of g-min-closed sets are independent in each other. See the following two examples:

Example 4.5. In Example 1.11, $\{b\}$ is g-mr closed, but not g-min-closed.

Example 4.6. Let $X=\{1,2,3,4\}$ with the topology $\tau=\{\phi, X,\{1\},\{4\},\{1,4\},\{1,2\},\{1,2,4\},\{1,3,4\}\}$. Then, the set $\{1,2\}$ is g-min-closed, but not g-mr closed.

Theorem 4.7. Let $X$ be a topological space. If $A$ is a nonempty min-g-closed set, then $A$ is $m r$-g-closed.

Proof. Since $A$ is min-g-closed, then there exists a minimal open set $U$ such that $C l(A) \subseteq U$. Since $\phi \neq A \subseteq U$, By Theorem 1.5, $C l(A)=C l(U)$. If $C l(U)=X$, then $X=$ $C l(A) \subseteq U \neq X$ which is a contradiction. So, $U$ is not dense is $X$. This implies that, by Theorem 2.1, $\operatorname{Int}(C l(U))$ is a minimal regular open and we have $A \subseteq U \subseteq \operatorname{Int}(C l(U))$. Hence $C l(A) \subseteq U \subseteq \operatorname{Int}(C l(U))$.

Remark 4.8. The converse of Theorem 4.7 need not be true.

Example 4.9. In Example 1.11, the set $\{b\}$ is $m r$-g-closed, but not min-g-closed.

Theorem 4.10. If $A$ is a mr-g-closed set, then $A$ is a regular g-closed.

Proof. Let $A \neq \phi$ be a mr-g-closed and $G$ a regular open set such that $A \subseteq G$. Let $U$ be a minimal regular open set such that $C l(A) \subseteq U$. Then $U \cap G \neq \phi$, so by Lemma 1.13, $U \subseteq G$. Thus, $C l(A) \subseteq G$.
Example 4.11. In Example 1.11, the set $\{c, d\}$ is a regular g-closed, but not mr-g-closed.

Theorem 4.12. Let $U$ be a minimal regular open set and $A \subseteq U$. Then, $A$ is a regular $g$-closed iff $A$ is an $m r$-g-closed.

Proof. Assume $A \neq \phi$ is a mr-g-closed, then by Theorem 4.10, $A$ is regular g-closed. Conversely, assume $A$ is regular g-closed. Since $A \subseteq U$ and $U$ is a minimal regular open set, $U$ is regular open such that $A \subseteq U$. Then $C l(A) \subseteq U$.

Theorem 4.13. Let $X$ be a semi-regular space and $A$ a subset of $X$. Then, $A$ is g-min-closed iff $A$ is g-min-regular closed.

Proof. Follows from Theorem 3.1.

Corollary 4.14. Let $\left(X, \tau_{s}\right)$ be the semi-regularization of a topological space $(X, \tau)$, then the following statements are equivalent:

(a) A is g-mr closed set in $(X, \tau)$.

(b) A is g-mr closed set in $\left(X, \tau_{s}\right)$.

(c) A is g-min-closed set in $\left(X, \tau_{s}\right)$.

Proof. Follows directly from Theorem 3.11 and Corollary 3.4 .

Theorem 4.15. Let $A$ be an mr-g-closed. If $A \subseteq B \subseteq$ $C l(A)$, then $B$ is $m r-g$-closed set.

Proof. Let $U$ be a minimal regular open set such that $C l(A) \subseteq U$. Then $C l(B) \subseteq C l(C l(A))=C l(A) \subseteq U$.

Theorem 4.16. Let $Y$ be an open subspace of $X$ and $G$ a minimal regular open set in $X$ such that $G \cap Y \neq \phi$ and $Y \nsubseteq G$. If $A \subseteq G$ is an mr-g-closed in $X$, then $A \cap Y$ is an mr-g-closed in $Y$.

Proof. By Theorem 2.9, $G \cap Y$ is minimal regular open in $Y$. $C l_{Y}(A \cap Y)=C l_{X}(A \cap Y) \cap Y \subseteq C l_{X}(A) \cap Y \subseteq G \cap Y$.

Theorem 4.17. Let $U$ be a minimal regular open set and $A \subseteq U$ such that $\operatorname{Int}(A) \neq \phi$. Then, $U$ is closed if and only if $A$ is regular $g$-closed.

Proof. If $U$ is closed, by Theorem 2.4, $C l(A)=C l(U)=$ $U$. Thus, by Theorem 4.10, $A$ is regular g-closed. Conversely, if $A$ is regular g-closed, by Theorem $2.4, C l(U)=$ $C l(A) \subseteq U$. Therefore, $U$ is closed.

Corollary 4.18. If $A$ is an $m r$-g-closed and closed with $\operatorname{Int}(A) \neq \phi$, then $A$ is both minimal regular open and minimal regular closed.

Proof. Let $U$ be a minimal regular open set such that $C l(A) \subseteq U$. Since $\operatorname{Int}(A) \neq \phi$, then by Theorem 2.4, $U \subseteq C l(A)$. This implies that $A=C l(A)=U$; that is, $A$ is minimal regular open and so, by Theorem $2.13, A$ is minimal regular closed. 


\section{$5 \quad r T_{\min }$ space}

Definition 5.1. [11] A topological space $(X, \tau)$ is said to be an $r T_{\min }$ space if every proper nonempty regular open subset of $X$ is minimal regular open.

Theorem 5.2. Let $(\mathrm{X}, \tau)$ be a topological space, then the following are equivalent:

(1) $X$ is $r T_{\min }$ space.

(2) Every proper nonempty regular open subset of $X$ is maximal regular open.

(3) Any pair of two distinct proper nonempty regular open sets are disjoint.

Proof. $(1 \Rightarrow 2)$ Let $U$ be a nonempty proper regular open. If $W$ is a proper regular open set such that $U \subseteq W$, then $W$ is minimal regular open. So by Lemma $1.13, W=U$. Thus $U$ is maximal regular open.

$(2 \Rightarrow 3)$ Let $U_{1}$ and $U_{2}$ be two proper nonempty regular open sets in $X$. Assume $U_{1} \cap U_{2} \neq \phi$, then $\phi \neq U_{1} \cap U_{2} \subseteq U_{1} \subset$ $X$; that is, $U_{1} \cap U_{2}$ is a proper nonempty regular open set and so $U_{1} \cap U_{2}$ is maximal regular open. Since $U_{1} \cap U_{2} \subseteq U_{1} \subset$ $X$ and $U_{1}$ is regular open, then $U_{1}=U_{1} \cap U_{2}$. Similarly, $U_{2}=U_{1} \cap U_{2}$. This implies that $U_{1}=U_{2}$.

$(3 \Rightarrow 1)$ Let $U$ be a proper nonempty regular open set and $W$ a nonempty regular open set such that $W \subseteq U \neq X$. So $W=U$. [Otherwise, $W \neq U$ and $W \cap U \neq \phi$ ]. Hence $U$ is a minimal regular open.

Corollary 5.3. Let $X$ be an $r T_{\min }$ space. Then, $m_{i} R O(X)=M_{a} R O(X)$.

Theorem 5.4. A nonempty open subspace $\left(A, \tau_{A}\right)$ of an $r T_{\text {min }}$ space $(X, \tau)$ is $r T_{\text {min }}$ space.

Proof. Let $U$ be a nonempty regular open set in $A$ such that $U \neq A$. By Theorem 1.2, there exists a nonempty proper regular open set $G$ in $X$ such that $U=G \cap A$. As the space $X$ is $r T_{m i n}, G$ is a minimal regular open in $X$. By Theorem 2.9, $U$ will be minimal regular open set in $A$.

\section{REFERENCES}

[1] A.S.Mashhour, M.E. Abd El-Monsef, and S.N. El-Deeb, On precontinuous and weak precontinuous mappings, Proc. Math. Phys.Soc. Egypt, 53, 47-53, (1982).

[2] C. Dorsett, New Characterization of Regularly Open Sets, Extremely Disconnected and RS-Compactness, J. Sci. Research, 8, 95-99, (1986).
[3] C. Dorsett, Regularly Open Sets and r-topological Properties, Nat. Acod. Sci. Letters, 10, 17-21, (1987).

[4] C. Dorsett, Regularly Open Sets, Regularly Closed Sets and New Characterization of Separation Axioms, Univ. J. Math. Sci, 6, 53-63, (2014).

[5] C. Dorsett, Regularly Open Sets, the Semi-regularization Process and Subspaces, Pioneer. J. Math. Sci, 12, 29-33, (2014).

[6] F. Nakaoka and N. Oda, Some application of minimal open sets, Int. J. Math. Sci, 27(8), 471-478, (2001).

[7] F. Nakaoka and N. Oda, Some properties of maximal open sets, Int. J. Math. Sci, 21, 1331-1340, (2003).

[8] J. Dontchev, Survey on preopen sets, The Proceedings of the Yatsushiro Topological conference, 1-18, (1998).

[9] M. Mrsevc, I. Reilly, and M. Vamanamurth, On Semiregularization Topologies, J. Austral. Math. Sci, 38, 40-54, (1985).

[10] M. Stone, Applications of the theory of Boolean rings to general topology. Trans. Amer. Math. soc, 41, 374-481, (1937).

[11] N. Anuradha and B. Chacko, On minimal regular open sets and maps in topological spaces, J. Math. Sci, 4, 182-192, (2015).

[12] N. Bourbaki, Elements of Mathematics, General Topology. (Herman Paris; Addison-Weakly, Reading, Mass), (1966).

[13] N. El-Deep, I.A. Hasanein, A.S. Mashhour, and T. Noiri, On p-regular spaces. Bull. Math. Soc. Sci. Math. R.S. Roumanie, 27(75), 311-315, (1983).

[14] N. Levine, Semi-open sets and semi-continuity in topological spaces, Amer. Math. Monthly,70, 36-41, (1963).

[15] N. Palaniappan and K.C. Rao, Regular Generalized Closed Sets, Kyungpook Math. J., 33, 211-219, (1993).

[16] O. Njastad, On some classes of nearly open sets, Pacific J. Math, 15, 961-970, (1965).

[17] S. Benchalli, S. Banasode, and G. Sidddapur, Generalized Minimal Closed Sets in Topological Spaces, J. Comp. Math. Sci, 6, 710-715, (2010).

[18] S. Bhattacharya, On Generalized Minimal Closed Sets, Int. J. Contemp. Math. Sci, 6, 153-160,(2011).

[19] S.G. Crossley and S.K. Hildebrand, Semi-closure, Texas. J. Sci, 22, 99-112, (1971).

[20] T.H. Jasim and N.I. Aziz, On Generalized Minimal Open Set and Some Properties, Australian. J. Basic and Applied Sci, 8, 24-36, (2014). 